\title{
Analysis of RCS of Low Observable Aircraft in VHF Band
}

\author{
Yi-Ru Jeong $\mathbb{D},{ }^{1}$ Chan-Sun Park, ${ }^{1}$ Young-Kwan Ko, ${ }^{2}$ and Jong-Gwan Yook $\mathbb{D}^{1}$ \\ ${ }^{1}$ School of Electrical and Electronic Engineering, Yonsei University, Seoul 120-749, Republic of Korea \\ ${ }^{2}$ Hanwha Systems, Yongin, Republic of Korea \\ Correspondence should be addressed to Jong-Gwan Yook; jgyook@yonsei.ac.kr
}

Received 17 October 2017; Revised 6 February 2018; Accepted 4 March 2018; Published 29 April 2018

Academic Editor: Atsushi Mase

Copyright (C) 2018 Yi-Ru Jeong et al. This is an open access article distributed under the Creative Commons Attribution License, which permits unrestricted use, distribution, and reproduction in any medium, provided the original work is properly cited.

Electromagnetic signatures of a low observable aircraft have been studied in VHF band. First of all, a three-dimensional model of the aircraft has been established for numerical computation. Then, monostatic and bistatic radar cross sections (RCS) have been calculated. The model of the aircraft is made by a curved surface, and commercial as well as in-house three-dimensional electromagnetic code which is based on the method of moments (MoM) is utilized to calculate the RCS. A characteristic basis function method (CBFM) and a multilevel fast multipole algorithm (MLFMA) have been applied to analyze electrically large objects. The change of the monostatic RCS is very large depending on the direction of the incident wave. The maximum value is about $42 \mathrm{dBsm}$ at the top and bottom of the aircraft, and the minimum value is about $-10 \mathrm{dBsm}$ at the front and back of the aircraft. It is found that the bistatic RCS also changes dramatically depending on the direction of the incident wave. The direction of maximum RCS occurs around specular reflection, and the value of maximum RCS ranges from $27 \mathrm{dBsm}$ to $43 \mathrm{dBsm}$. On the other hand, the direction of the minimum RCS occurs irregularly, and the value is in the level of $-30 \mathrm{dBsm}$.

\section{Introduction}

Various low observable technologies for an aircraft have been utilized to reduce radar cross section (RCS) to avoid detection by radars and have become a vital design goal of a tacti$\mathrm{cal}$ aircraft. The shape of the aircraft is the most important factor, and in general, low observable performance is targeted at a high frequency region, such as the $\mathrm{X}$ band [1]. On the other hand, preliminary researches on the detection of a low RCS aircraft using the radars in a low frequency region have been attempted [2-4]. In the low frequency, radars can be designed to be installed on the ground or on sea vessels since the size of radar is relatively larger compared to a high frequency system.

Based on a radar equation, the received power is proportional to the square of the wavelength if all other parameters are fixed. Thus, it is clear that using lower frequency is an effective way of detecting long range target or low RCS objects if the RCS is theoretically known at that frequency region. Of course, one needs a larger antenna size to have a similar gain of the higher frequency system. On the other hand, the size of a modern low observable aircraft is comparable to the wavelength in VHF and UHF regions and the RCS will be in the resonant region, rendering effective detection of objects in the far distance. In this paper, RCS of a low observable aircraft in the VHF band is calculated using rigorous numerical techniques, and variation of RCS depending on the polarization and direction of the incident wave is analyzed. Numerical techniques and model for an aircraft are important to accurately predict RCS. Thus, a three-dimensional aircraft model is built using the curved surface to reduce computational cost and improve the accuracy of the result. Then, RCS is calculated using the aircraft model in the VHF band. In-house code and commercial software which are based on the method of moments (MoM) are used to calculate RCS. Although the MoM is a well-known full-wave method which is capable of predicting the RCS of arbitrary conducting objects, the method itself is very computationally expensive and sometimes not possible for electrically large structures. Thus, a characteristic basis function method (CBFM) [5-7] is applied to in-house MoM code, while a multilevel fast multipole algorithm (MLFMA) [8] which is built in the commercial software [9] is utilized. Monostatic RCS of the low observable aircraft in VHF is 


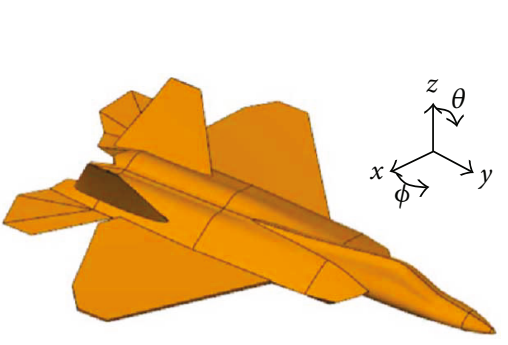

(a)

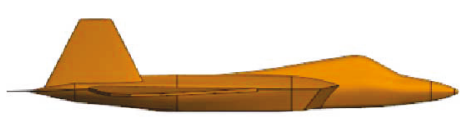

(c)

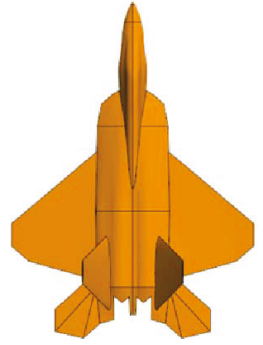

(b)

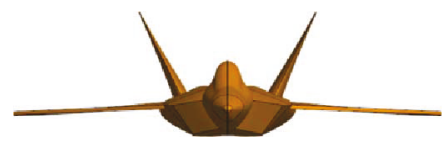

(d)

FIGURE 1: Overview of an aircraft.

calculated, and the change of RCS depending on the direction and the polarization of the incident wave is analyzed. Also, after bistatic RCS is obtained as a function of the angle of incidence and polarization of the incident wave, the direction of the maximum as well as the minimum RCS has been found.

\section{Modeling of the Aircraft}

The model of the aircraft in this paper is based on F-22. Many types of radars for detecting an aircraft are developed in high frequency such as the $\mathrm{X}$ band. Then, the aircraft is generally designed to minimize RCS in high frequency than low frequency. In this situation, radar in low frequency such as the VHF band is useful although it has disadvantages in terms of size and installation. The RCS of the aircraft which is not optimized in the VHF band is analyzed using this model.

Reliability and accuracy of the calculated RCS depend on the accuracy of the three-dimensional electromagnetic model of the aircraft. In order to enhance the accuracy of the model, the structural details and surface curvatures of the aircraft have to be reflected in the model as much as possible. However, computational resources, such as calculation time and computer memory, increase exponentially with the addition of small structures. Therefore, compromise between accuracy and efficiency is important in the modeling of an aircraft. The model which is used in this paper is shown in Figure 1. It should be noted that the external surface of the aircraft is represented as the perfect electric conductor (PEC), and the interior details, such as an engine and air intake, are omitted to reduce overall computational cost at the expense of degraded accuracy.

The engine inlet of the aircraft model in this paper is closed. Although an engine inlet should be included to obtain more accurate RCS, RCS in a high frequency region such as the $\mathrm{X}$ band has been reported in most of the papers about engine inlet or open-ended cavity. Electromagnetic characteristics are different between high and low frequencies. For example, interior irradiation is much larger than the rim diffraction, especially high frequency. Also, the nose, inlets, nozzle, and junctions between the fuselage, the wings, and the

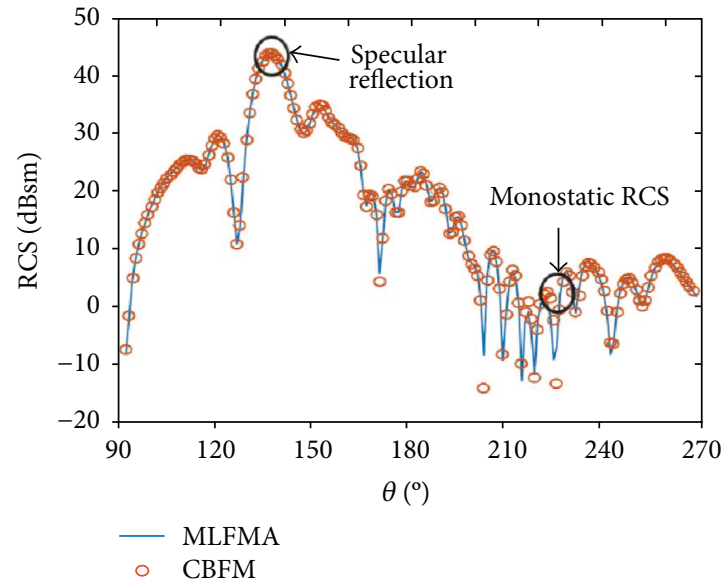

Figure 2: Bistatic RCS of an aircraft.

stabs will present as Raleigh regime scattering centres. Therefore, the analysis of structures such as the engine inlet should be done carefully in the VHF band. The analysis of the RCS of the engine inlet in VHF will be future work.

The model comprises 66 curved surfaces, resulting in about 60,000 triangular meshes at $200 \mathrm{MHz}$.

\section{Numerical Method}

In the conventional MoM, the electromagnetic scattering problem with PEC in a free space is dealt with the electric field integral equation (EFIE), and surface current is expressed by the Rao-Wilton-Glisson (RWG) basis function. However, in order to analyze an electrically large object, such as an aircraft, the utilization of CBFM and MLFMA would be necessary.

Each acceleration technique has advantages. For the same geometry, if the frequency is fixed, excitation is changed; CBFM is more efficient than MLFMA because the impedance matrix for direct solver is constructed. In this paper, monostatic and bistatic RCS is analyzed depending on the polarization and direction of the incident wave in a frequency. Therefore, CBFM is used for obtaining the RCS of the aircraft 


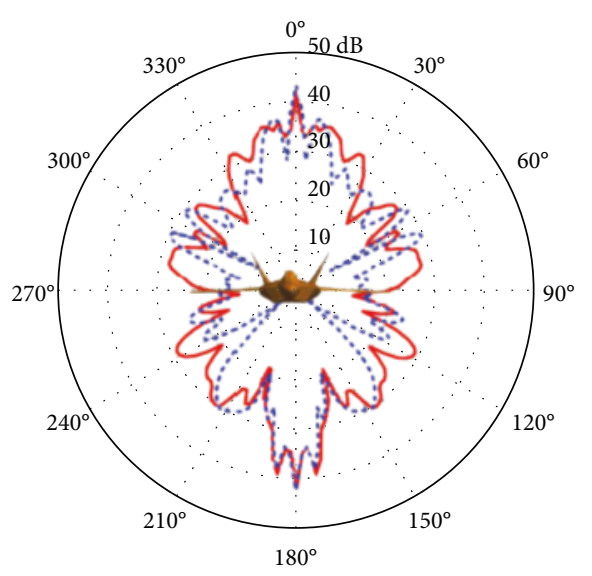

$\begin{array}{ll}- & \theta \theta \\ \cdots \cdots & \phi \phi\end{array}$

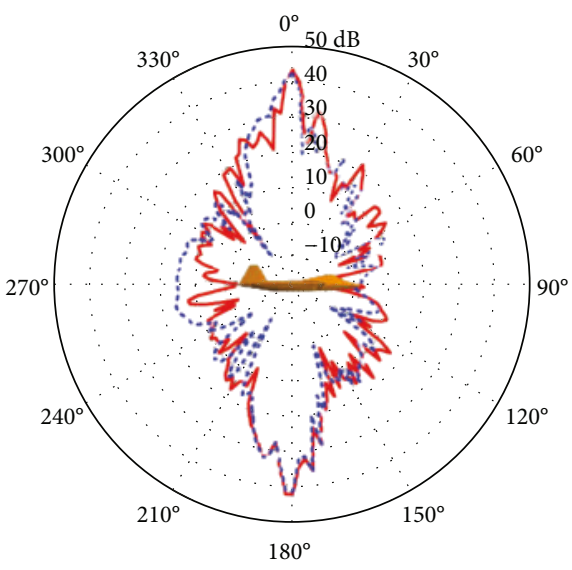

$-\theta \theta$

(a)

(b)

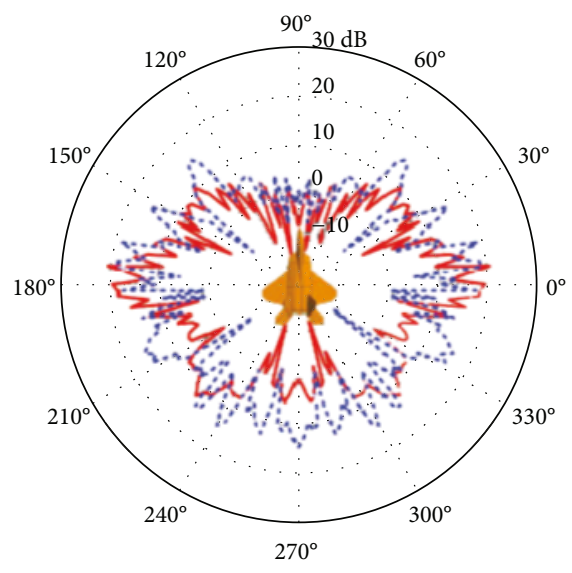

(c)

Figure 3: Monostatic RCS of an aircraft for copolarization. (a) $x z$ plane. (b) $y z$ plane. (c) $x y$ plane.

in this paper. In most commercial software, MLFMA is applied. Thus, commercial software using MLFMA is used to verify the accuracy of the in-house code based on CBFM.

3.1. Characteristic Basis Function Method (CBFM). In-house MoM code based on CBFM is used to analyze the full-size aircraft. The CBFM starts with dividing original geometry into several blocks to construct characteristic basis (CB). In this stage, the divided blocks do not overlap each other. And then, every block is extended into the region beyond the original boundary as much as 0.2 to $0.6 \lambda$ to avoid singularity of the current distribution at the boundary. The geometry in each block is regarded as a single entity when CBs are calculated. And, for each block, 800 external plane waves are illuminated. The interval between the plane waves is $20^{\circ}$ in azimuth and elevation, and vertical as well as horizontal polarizations of the plane wave are employed. Then, 800 matrixes of which the size is the number of unknowns in each block are obtained as the illumination matrixes. The linear equation can be derived with the impedance matrix in the block and illumination matrix. When the linear equation is solved, the current distribution is obtained as original CBs from 800 excitation vectors. However, all $800 \mathrm{CBs}$ are not necessary to represent current distribution in the block. Thus, singular value decomposition (SVD) is applied to eliminate redundancy, resulting in computational efficiency. Note that the diagonal elements indicate the representativeness of each basis function. In this letter, CBs which have a singular value less than the threshold have been eliminated from the $\mathrm{CB}$ group, where the threshold has been set to 0.001 of the maximum singular value. The remaining bases are taken as CBs for each block. By applying the SVD technique, the redundant basis has been removed and the size of the matrix is greatly reduced. The matrix is treated as the $\mathrm{CB}$ matrix for each block.

When CB generation for each block is finished, the process that combines block information to one reduced matrix is required. The size of the reduced linear system is much smaller compared to the original geometry problem. Thus, it can be solved with a direct solver. By reallocating each unknown to the original low-level basis index, the current density or RCS can be obtained. 


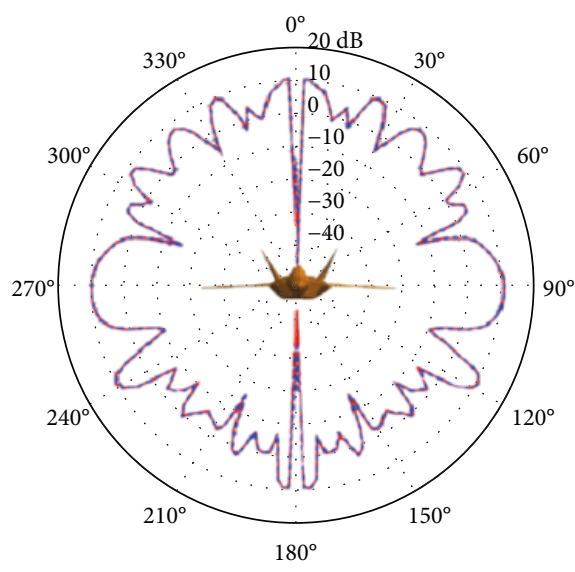

$-\theta \phi$

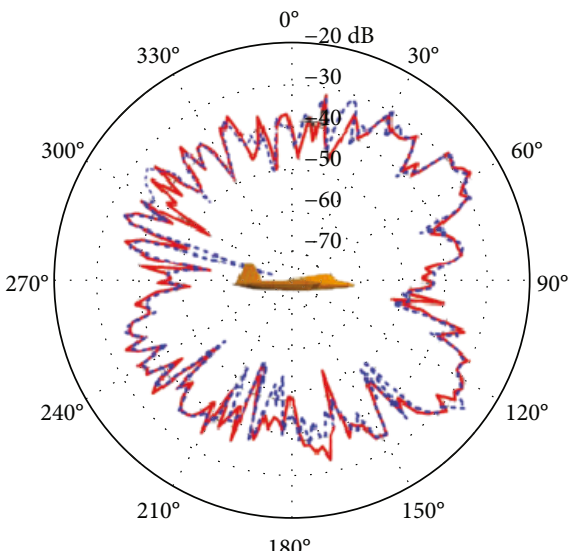

$-\theta \phi$

(a)

(b)

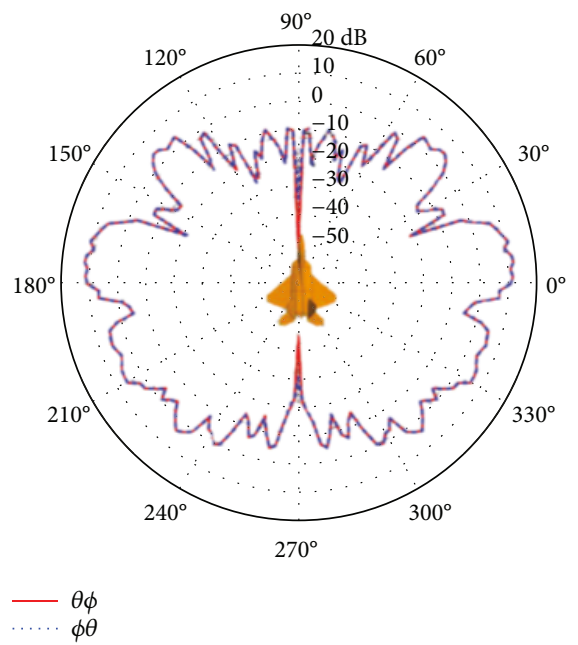

(c)

Figure 4: Monostatic RCS of an aircraft for cross polarization. (a) $x z$ plane. (b) $y z$ plane. (c) $x y$ plane.

3.2. Comparison of RCS between CBFM and MLFMA. As shown in Figure 1, there is an aircraft on the $x y$ plane. The cockpit is located towards the $+y$ direction, and the main wings are located along the $\pm x$ direction. The plane wave is incident at $\phi^{i}=0^{\circ}$ and $\theta^{i}=225^{\circ}$, and the frequency is $230 \mathrm{MHz}$. As shown in Figure 2, RCS using CBFM is similar with that using MLFMA. RCS is the maximum in the direction of specular reflection. Thus, CBFM is used for other numerical results in this paper.

\section{Monostatic RCS}

4.1. PEC. As shown in Figure 1, there is an aircraft on the $x y$ plane. The cockpit is located towards the $+y$ direction, and the main wings are located along the $\pm x$ direction. When the incident wave is $230 \mathrm{MHz}$, the monostatic RCS for the aircraft is analyzed. The monostatic RCS in the $x y$ plane, $y z$ plane, and $x z$ plane is calculated depending on the polarization and the direction of the incident wave. When the incident wave is copolarization, RCS is shown in Figure 3, where $\theta \theta$ means that the incident wave has $\theta$ direction and the scattered wave for the $\theta$ direction is also calculated and $\phi \phi$ means that the incident wave has $\phi$ direction and the scattered wave for the $\phi$ direction is also calculated.

When $\phi^{i}$ is $90^{\circ}$ and $\theta^{i}$ is $0^{\circ} \sim 360^{\circ}$, the monostatic RCS is shown in Figure 3(a). When the top and bottom of the aircraft are observed, the RCS becomes very high and the value is about $40 \mathrm{dBsm}$. On the contrary, the side of the aircraft is observed, the RCS becomes small, and the value is about $8 \mathrm{dBsm}$. When polarization is changed from $\theta \theta$ to $\phi \phi$, the RCS is also changed. Particularly, when the aircraft is observed obliquely, the change of the RCS is serious. Even if the overall tendency is similar between $\theta \theta$ and $\phi \phi$, the specific value is changed depending on the direction of the incident wave. When the direction of the incident wave is $\theta=0^{\circ} \sim 360^{\circ}$ and $\phi=0^{\circ}$, the monostatic RCS is shown in Figure 3(b). The RCS for the top and bottom of the aircraft is similar between Figures 3(a) and 3(b). When the front and back of the aircraft are observed, the RCS is very small and the specific value is about $-10 \mathrm{dBsm}$, Therefore, when the radar for detecting a low observable aircraft is designed, it is important to know this tendency for the RCS because 


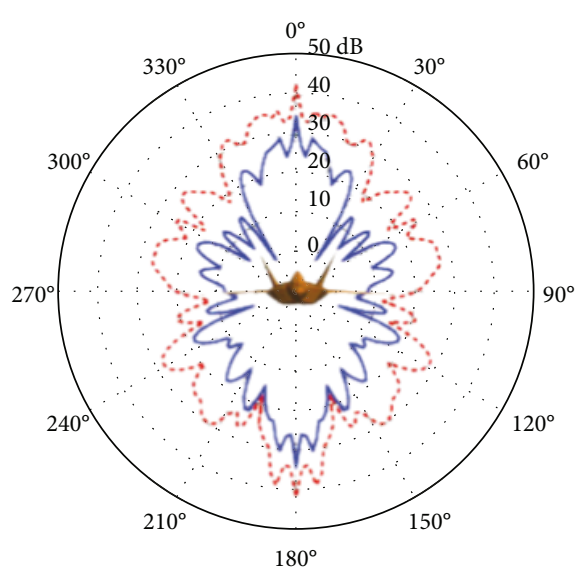

$\cdots$ PEC
- PEC-TDS

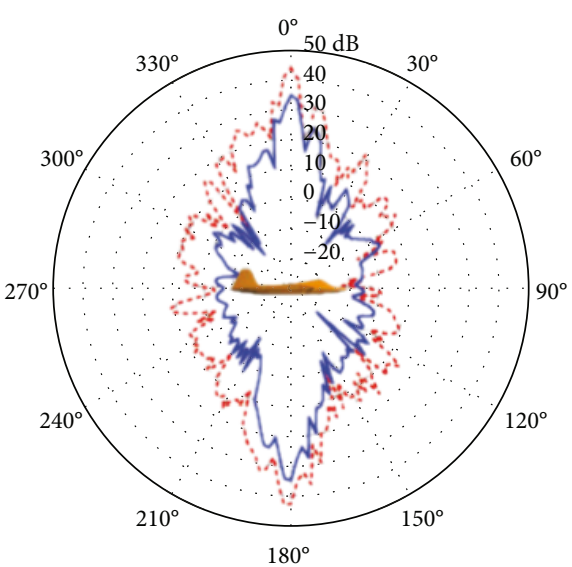

PEC

- PEC-TDS

(a) (b)

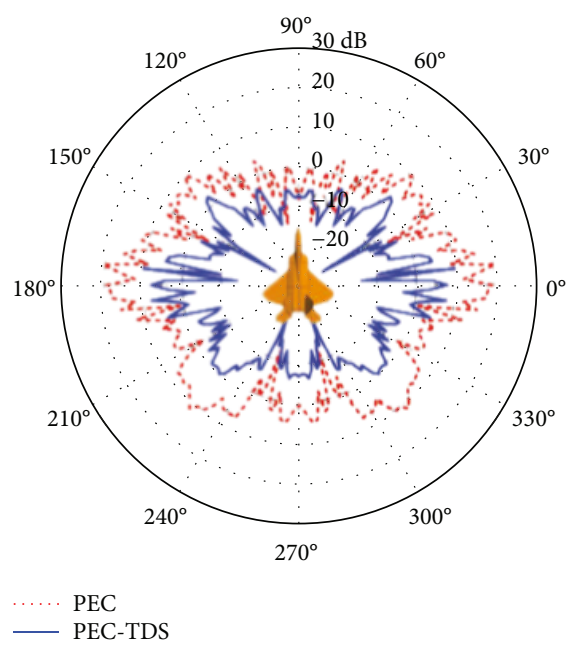

(c)

Figure 5: Monostatic RCS of a dielectric coated aircraft for theta-polarization. (a) $x z$ plane. (b) $y z$ plane. (c) $x y$ plane.

the change of the RCS depending on the direction of the incident wave is severe. When the direction of the incident wave is $\theta=90^{\circ}$ and $\phi=0^{\circ} \sim 360^{\circ}$, the monostatic RCS is shown in Figure 3(c). The RCS in Figure 3(c) is generally smaller than that in Figures 3(a) and 3(b) because the side of the aircraft is observed. The RCS in the front and back of the aircraft is smaller than that in the side of the aircraft. The RCS for $\theta \theta$ is generally larger than that for $\phi \phi$ because $\theta \theta$ polarization is the direction of the front and back of the aircraft and $\phi \phi$ polarization is the direction of the top and bottom of the aircraft. Monostatic RCS for cross polarization is shown in Figure 4 . When the direction of the incident wave is $\theta=0^{\circ} \sim 360^{\circ}$ and $\phi=90^{\circ}$, the monostatic RCS is shown in Figure 4(a). $\theta \phi$ means that the direction of electric fields in an incident wave is $\theta$ and the scattered wave for the $\phi$ direction is calculated. In this way, $\phi \theta$ means that the direction of electric fields in an incident wave is $\phi$ and the scattered wave for the $\theta$ direction is calculated. The direction of the incident wave in Figure 4 (b) is $\theta=0^{\circ} \sim 360^{\circ}$ and $\phi=0^{\circ}$, and the direction of the incident wave in Figure 4(c) is $\theta=$ $90^{\circ}$ and $\phi=0^{\circ} \sim 360^{\circ}$. The RCS for cross polarization is much smaller than that for copolarization as well as the size of the aircraft. While the RCS in Figure 4(a) is larger than that in Figures 4(b) and 4(c), it has a small value compared with that in Figure 4(a) where the plane is the same with that in Figure 3(a). Also, the RCS between $\theta \phi$ and $\phi \theta$ is similar.

4.2. PEC with Dielectric Coating. An absorbing material layer such as RAS or RAM is essential to decrease RCS. While electromagnetic characteristics such as the reduction of the RCS and frequency band are the most important, mechanical characteristics such as thickness and durability for a severe environment have to be considered carefully. Thus, the design for realistic structures is complex. However, a simple absorber design is used in this paper because only the reduction effect of RCS is analyzed in the VHF band and mechanical characteristics are not considered. It is assumed that the ferrite material of which relative permittivity and permeability are 12.5 and $1.8-\mathrm{j} 29.3$, respectively, was used. When the thickness of the dielectric layer on planar PEC is $7 \mathrm{~mm}$, the reflection coefficient is $-27(\mathrm{~dB})$ at $230 \mathrm{MHz}$. Various numerical techniques have been developed to analyze the thin 


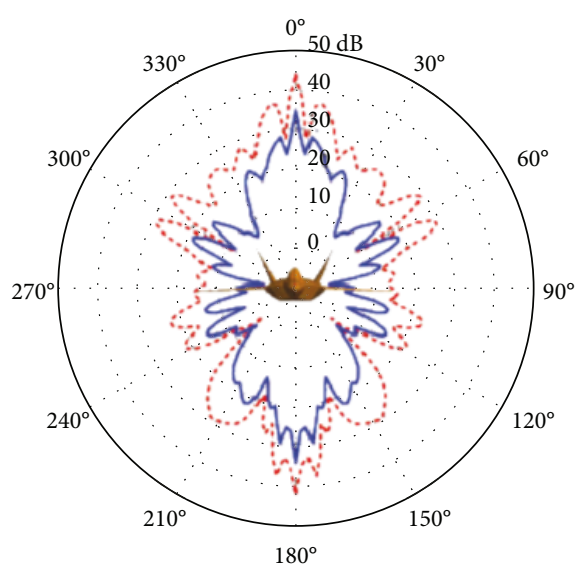

PEC — PEC-TDS

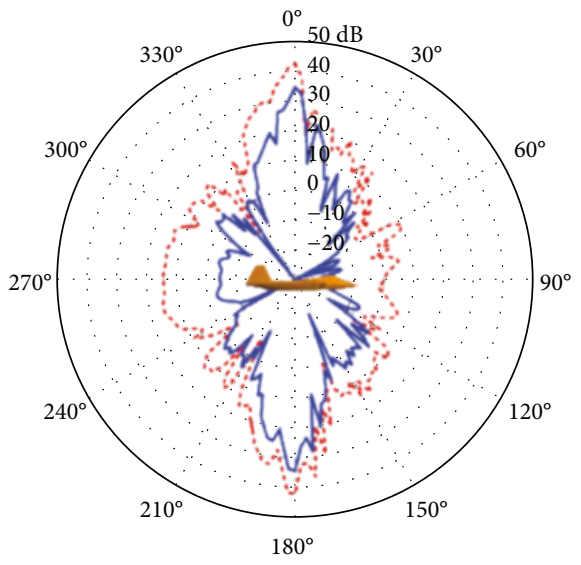

PEC

- PEC-TDS

(a)

(b)

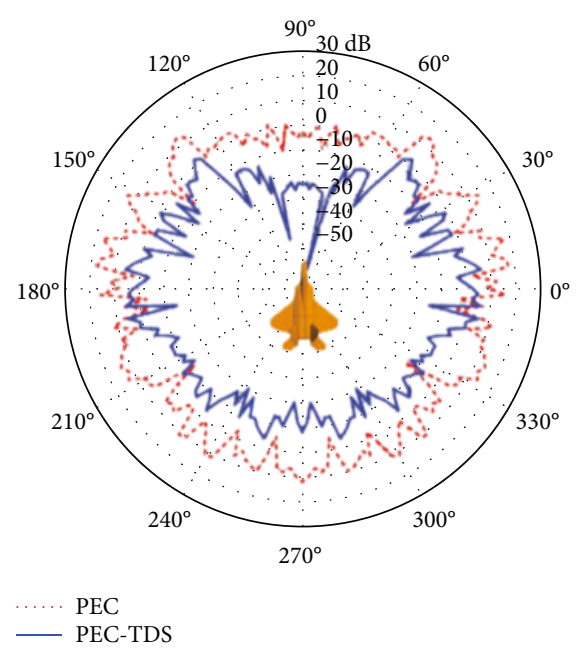

(c)

FIgURE 6: Monostatic RCS of a dielectric coated aircraft for phi-polarization. (a) $x z$ plane. (b) $y z$ plane. (c) $x y$ plane.

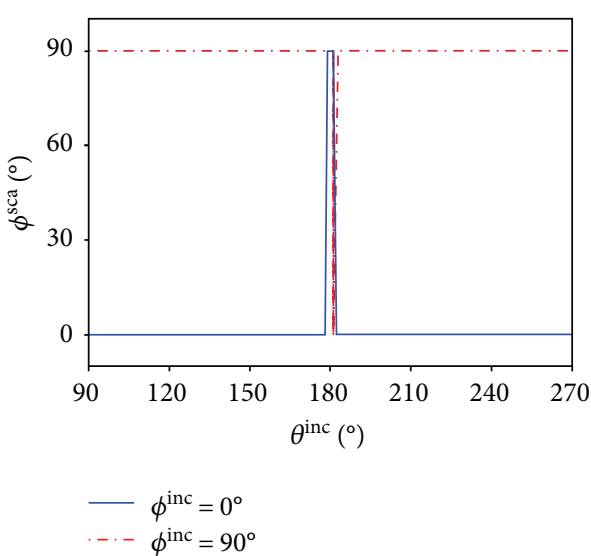

(a)

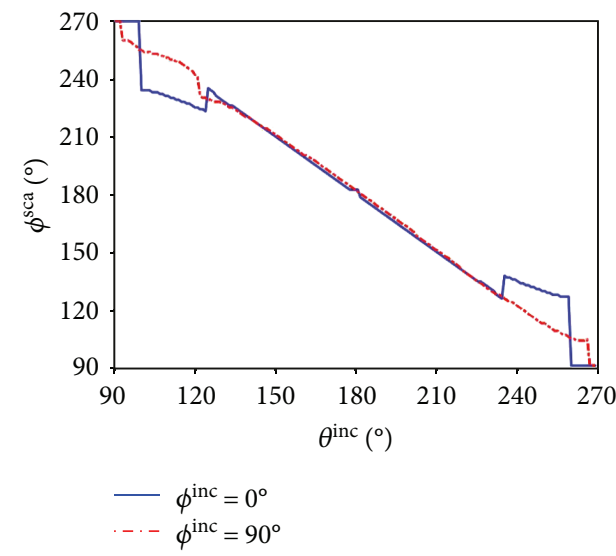

(b)

Figure 7: Direction of the maximum RCS $(\alpha=0)$.

dielectric layer on PEC. In this paper, thin dielectric sheet (TDS) approximation is used. In TDS, computational cost is reduced because the dielectric layer is approximated to the equivalent sheet by neglecting the change of the internal field along normal direction and considering only the tangential field along the thin dielectric layer. 


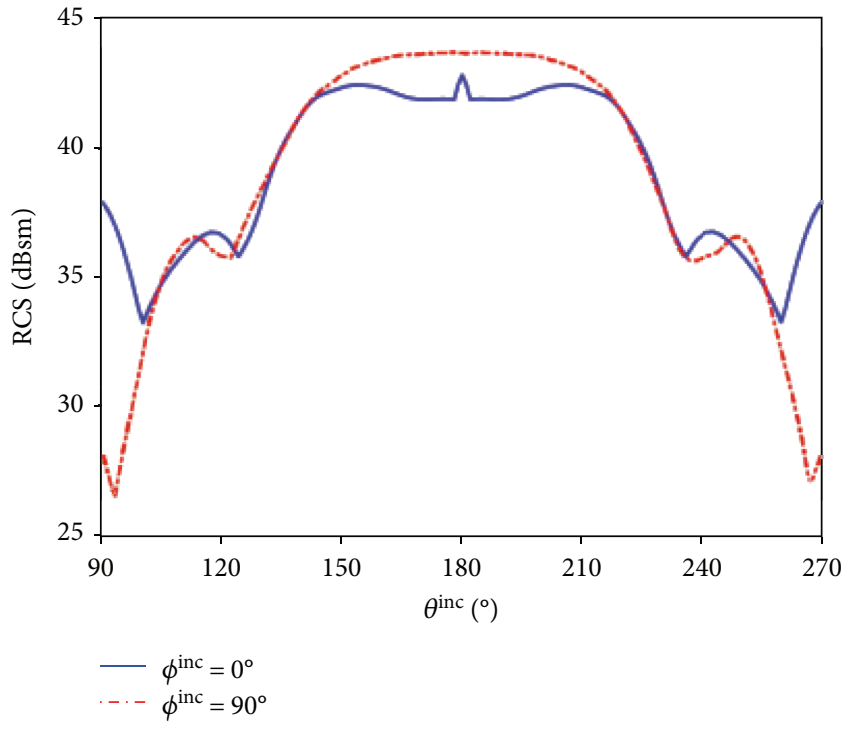

Figure 8: Value of the maximum RCS $(\alpha=0)$.

When the dielectric layer is applied to the aircraft, the monostatic RCS is shown in Figures 5 and 6. While the $\theta$ direction of the incident and scattered waves is considered in Figure 5, the $\phi$ direction of the incident and scattered waves is considered in Figure 6. The RCS of all directions is reduced by the absorbing material as shown in Figures 5 and 6. In Figure 5, RCS decreases as much as $5 \mathrm{dBsm}$ and $8 \mathrm{dBsm}$ in each direction of the front and back of the aircraft, and the reduction of RCS is about $10 \mathrm{dBsm}$ at the bottom of the aircraft. In Figure 6, RCS decreases as much as $20 \mathrm{dBsm}$ in the direction of the front and back of the aircraft, and the reduction of RCS is about $7 \mathrm{dBsm}$ at the bottom of the aircraft. Although monostatic RCS generally decreases, the reduction effect by the absorbing material is changed depending on polarization and direction. Also, the decrease of RCS in the aircraft is smaller than that in the planar structure because the reflection coefficient of the absorbing material varies with characteristics of the incident wave. Thus, when RAS or RAM is designed in the VHF band, it is important to maintain the reflection coefficient even if the incident wave or frequency is changed.

\section{Bistatic RCS}

The interval of the incident and scattered waves is $1^{\circ}$. Now, the incident plane wave is given as

$$
\mathbf{E}^{i}=\left(\widehat{x} E_{x}+\hat{y} E_{y}+\widehat{z} E_{z}\right) e^{j\left(k_{x} x+k_{y} y+k_{z} z\right)},
$$

where

$$
\begin{aligned}
& E_{x}=\cos \theta^{i} \cos \phi^{i} \cos \alpha-\sin \phi^{i} \sin \alpha, \\
& E_{y}=\cos \theta^{i} \sin \phi^{i} \cos \alpha+\cos \phi^{i} \sin \alpha, \\
& E_{z}=-\sin \theta^{i} \cos \alpha .
\end{aligned}
$$

where $\alpha$ represents two orthogonal polarizations; that is, $\alpha=0^{\circ}$ case does have no $z$-directed electric field component.

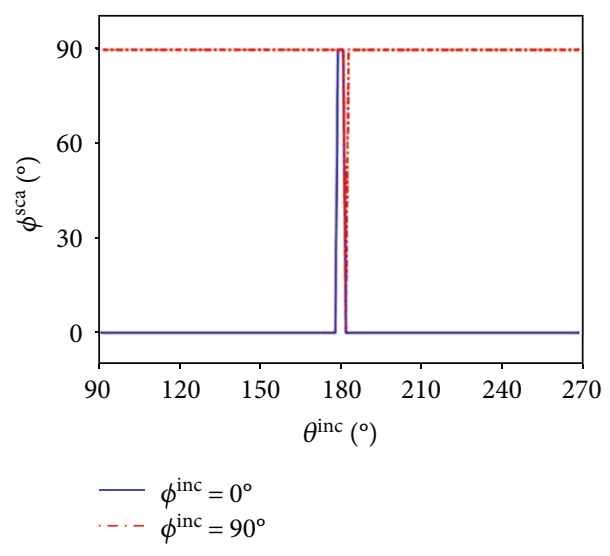

(a)

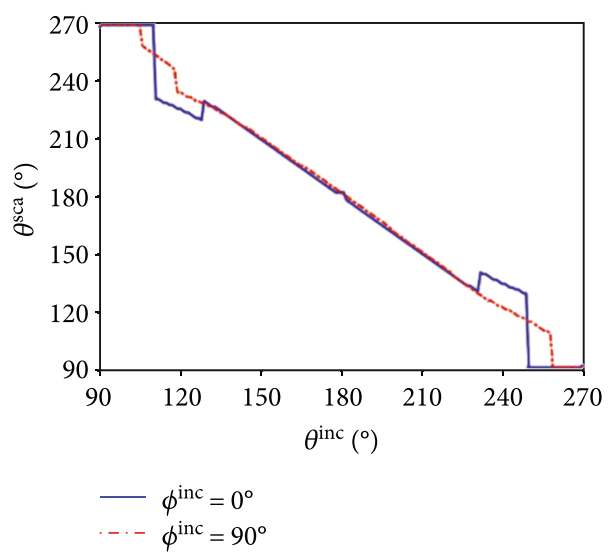

(b)

Figure 9: Direction of the maximum RCS $(\alpha=90)$.

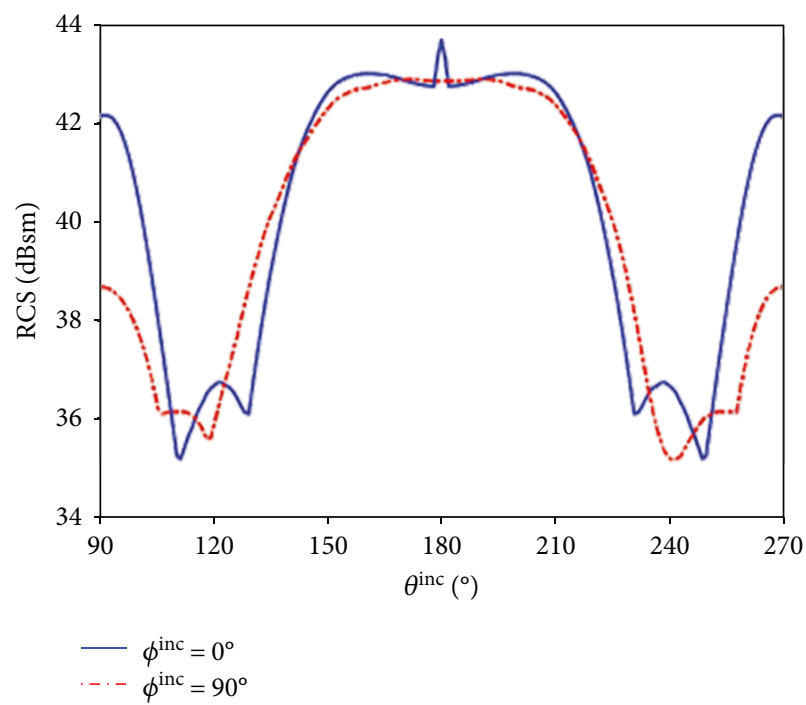

Figure 10: Value of the maximum RCS $(\alpha=90)$.

On the other hand, when $\alpha=90^{\circ}$, the electric field has $z$ component.

When the plane wave is incident upon the aircraft from the lower front side $\left(\phi^{i}=90^{\circ}, \theta^{i}=225^{\circ}\right)$, the bistatic RCS of 


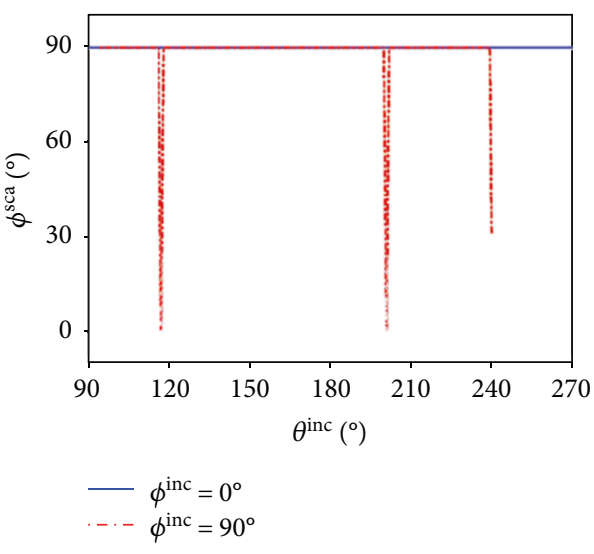

(a)

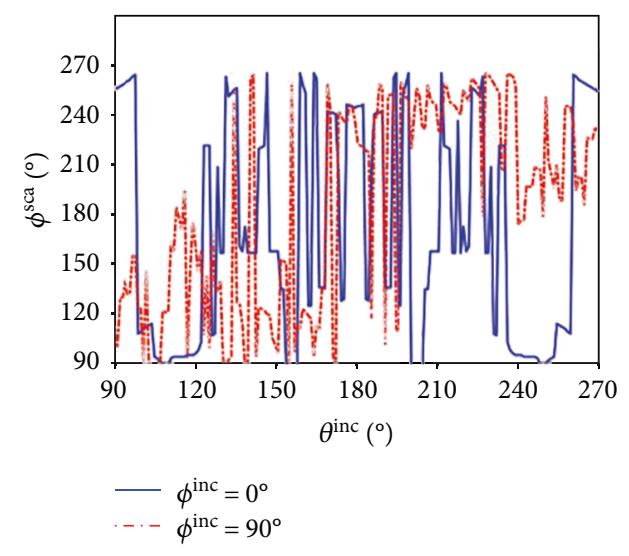

(b)

Figure 11: Direction of the minimum RCS $(\alpha=0)$.

the aircraft at $230 \mathrm{MHz}$ is calculated using the CBFM as well as the MLFMA as shown in Figure 2. The result from the MLFMA agrees very well with that of the CBFM. Monostatic $\operatorname{RCS}$ at $\left(\phi^{s}=90^{\circ}, \theta^{s}=225^{\circ}\right)$ is around $5 \mathrm{dBsm}$, and the fluctuation of RCS is huge which is greater than $50 \mathrm{dBsm}$. It has been observed that the maximum RCS value is about $42 \mathrm{dBsm}$ at the specular reflection detection $\left(\phi^{s}=90^{\circ}, \theta^{s}=\right.$ $135^{\circ}$ ) and the RCS changes rather slowly in the nearspecular reflection direction. Bistatic RCS can be calculated for any incident angle and observation direction. In this work, however, bistatic RCS is calculated with the incident angle from the lower side of the aircraft $\left(90^{\circ}<\theta^{i}<270^{\circ}\right)$ and the observation angle is also considered in the lower region $\left(90^{\circ}<\theta^{s}<270^{\circ}\right)$.

5.1. Maximum RCS. In this section, the direction of the maximum RCS has been found for various incident angles ranging from $\theta^{i}=90^{\circ}$ to $\theta^{i}=270^{\circ}$ with $\phi^{i}=0^{\circ}$ and $90^{\circ}$ When polarization $\alpha$ is 0 , the direction and value of the maximum RCS are shown in Figures 7 and 8 for two orthogonal planes $\left(\phi^{i}=0^{\circ}\right.$ and $\left.90^{\circ}\right)$. It is clear that most of the maximum values are observed at the specular directions. Of course, it is possible that the maximum RCS can occur at different planes due to the curved surface. It is interesting to observe that the maximum RCS for two orthogonal polarizations is greater than $35 \mathrm{dBsm}$ in the most incident angle; however, when $\alpha$ $=0^{\circ}$, that is, there is no $z$ component in the incident wave, the maximum RCS becomes less than $30 \mathrm{dBsm}$ around $\theta^{i}=$ $90^{\circ}$ and $270^{\circ}$. Also, it is clear that the maximum RCS is larger at $\phi^{i}=0^{\circ}$ than $\phi^{i}=90^{\circ}$ in most cases. Although the shape of the aircraft is for low observable performance, the maximum RCS is quite large near the specular reflection directions.

When polarization $\alpha$ is $90^{\circ}$, the direction and value of the maximum RCS are shown in Figures 9 and 10. The overall tendency in $\alpha=90^{\circ}$ is similar to that in $\alpha=0^{\circ}$. The direction of the maximum RCS is near to specular reflection regardless of polarization. When $\theta^{i}$ is $90^{\circ}$ and $270^{\circ}, \operatorname{RCS}$ in $\alpha=0$ is larger than RCS in $\alpha=90^{\circ}$ because the direction of the electric field in $\left(\alpha=0^{\circ}\right)$ is along the width direction of the aircraft.

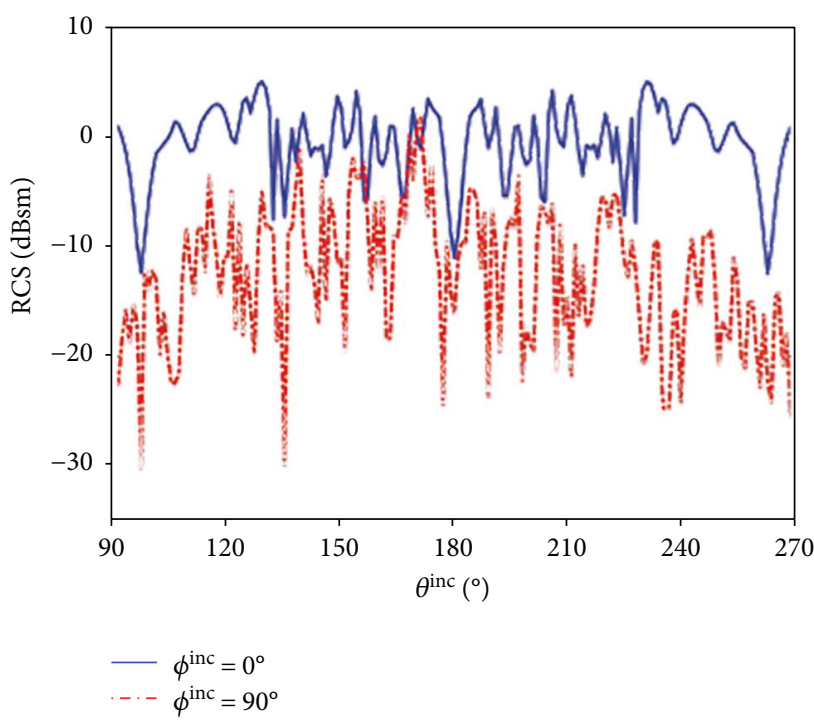

FIgURE 12: Value of the minimum RCS $(\alpha=0)$.

5.2. Minimum RCS. The minimum of RCS is found depending on the change of the incident direction. When polarization $\alpha$ is 0 , the direction and value of the minimum RCS are shown in Figures 11 and 12. Contrary to the maximum RCS, the direction of the minimum RCS is irregular. Particularly, there is no relation in the $\theta$ direction between the incident wave and the scattered wave where RCS is minimum. In the $\phi$ direction, $\phi^{s}$ of the minimum RCS is almost 90 because the effective area in $\phi=90^{\circ}$ is generally smaller than that in $\phi=0^{\circ}$ because the cockpit or tail is seen at $\phi=90^{\circ}$. When the $\phi^{i}$ direction is 0 , the range of the minimum RCS is from $5 \mathrm{dBsm}$ to $-12 \mathrm{dBsm}$. When the $\phi^{i}$ direction is 90 , the range of the minimum RCS is from $2 \mathrm{dBsm}$ to $-30 \mathrm{dBsm}$. The value of the minimum RCS is generally small and fluctuating sharply. Thus, it is difficult to detect the aircraft in this direction. The minimum RCS in $\phi^{i}=90$ is generally smaller than that in $\phi^{i}=0$ due to the difference of the effective areas.

When polarization $\alpha$ is 90 , the direction and value of the minimum RCS are shown in Figures 13 and 14. The overall 


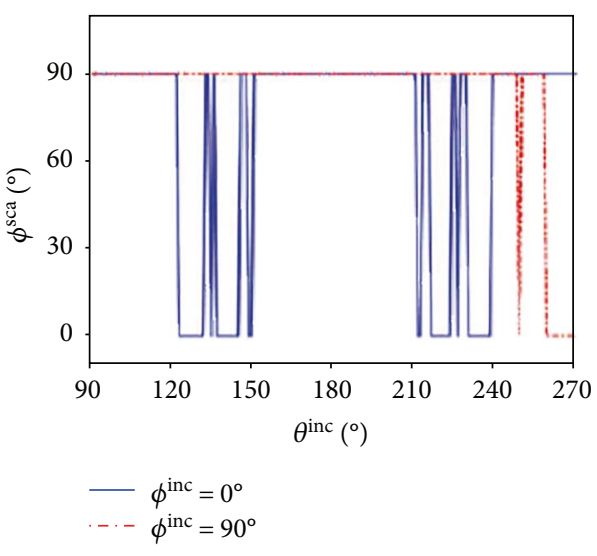

(a)

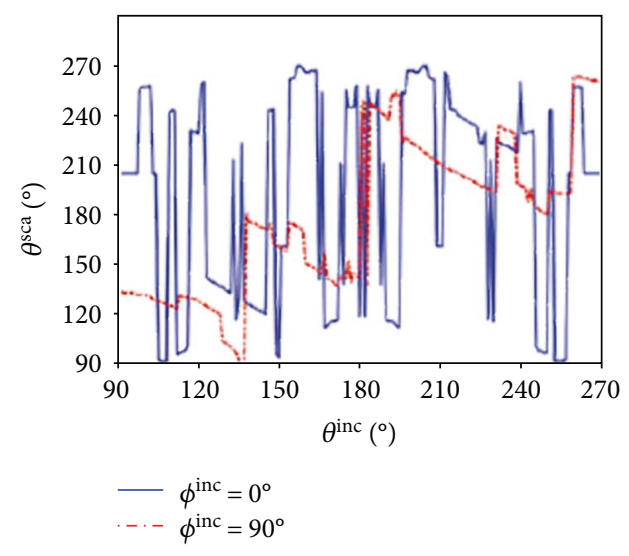

(b)

Figure 13: Direction of the minimum RCS $(\alpha=90)$.

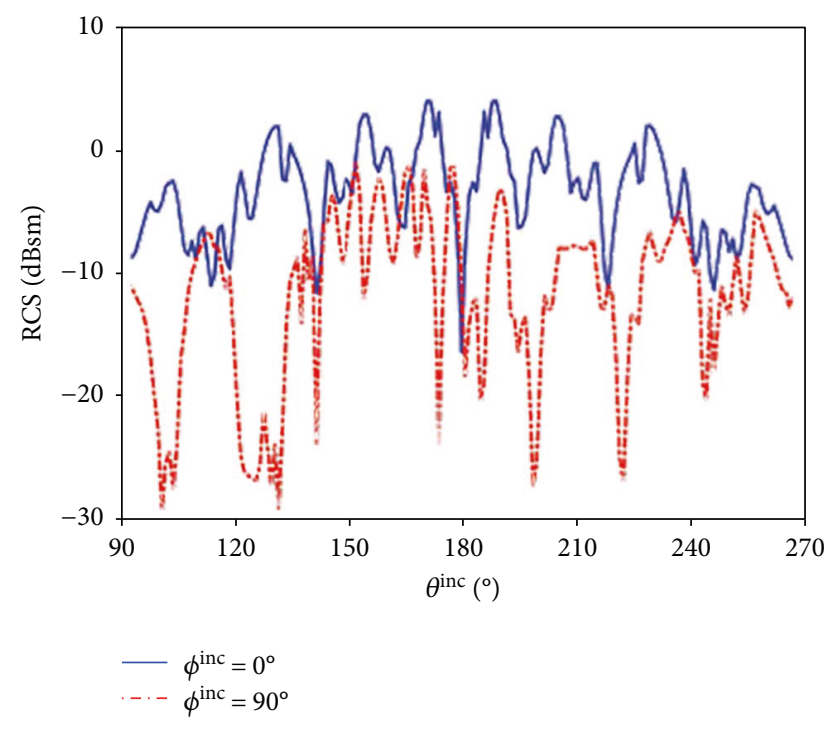

FIgURE 14: Value of the minimum RCS $(\alpha=90)$.

tendency in the case that $\alpha$ is 90 is similar to that in the case that $\alpha$ is 0 . The direction of the minimum RCS is also irregular. However, when $\phi^{i}$ is 90 , that is, the incident wave is from the head to the tail of the aircraft, the direction of the minimum RCS is near the incident direction. The value of the minimum RCS is from $3 \mathrm{dBsm}$ to $-30 \mathrm{dBsm}$. When $\theta^{i}$ is 90 and 270, RCS in $\alpha=0$ is larger than that in $\alpha=90$ because the direction of the electric field is along the width direction.

\section{Conclusion}

Researches for detecting the aircraft using radar in the VHF band are studied. Thus, the analysis of the RCS of the aircraft is required to design efficiently the radar. The range of monostatic RCS is very wide according to the direction of the incident wave. For copolarization, the maximum value is $42 \mathrm{dBsm}$, and the minimum value is $-10 \mathrm{dBsm}$. In addition, bistatic RCS is calculated. Maximum RCS occurs in the near to specular direction and is ranging from $44 \mathrm{dBsm}$ to $26 \mathrm{dBsm}$. Therefore, in order to detect the aircraft in the
VHF band, it is effective to use two radars and widen their space as much as possible. On the other hand, the direction of the minimum RCS is irregular, and its range is from $5 \mathrm{dBsm}$ to $-30 \mathrm{dBsm}$. The value of the minimum RCS is small, and its direction is roughly close to the incident direction. Unfortunately, it is difficult to find the accurate direction of the minimum RCS because RCS in the near to incident direction varies very sharply. It is expected that the results in this paper are of help to design the radar in the VHF band.

\section{Conflicts of Interest}

The authors declare that there are no conflicts of interest regarding the publication of this paper.

\section{Acknowledgments}

This work has been supported by research funds from Hanwha Systems.

\section{References}

[1] L. Gürel, H. Bağcı, J. C. Castelli, A. Cheraly, and F. Tardivel, "Validation through comparison: measurement and calculation of the bistatic radar cross section of a stealth target," Radio Science, vol. 38, no. 3, pp. 12-1-12-8, 2003.

[2] A. David, C. Brousseau, and A. Bourdillon, "Simulations and measurements of a radar cross section of a Boeing 747-200 in the 20-60 MHz frequency band," Radio Science, vol. 38, no. 4, 2003.

[3] C. Bares, C. Brousseau, and A. Bourdillon, "A multifrequency HF-VHF radar system for aircraft identification," in IEEE International Radar Conference, 2005, pp. 478-482, Arlington, VA, USA, May 2005.

[4] X. Fan, Y. Qin, S. Shang et al., "Research on the bastatic RCS characteristics of stealth aircraft," in 2015 Asia-Pacific Microwave Conference (APMC), pp. 1-3, Nanjing, China, December 2015.

[5] E. Lucente, A. Monorchio, and R. Mittra, "An iteration-free MoM approach based on excitation independent characteristic basis functions for solving large multiscale electromagnetic 
scattering problems," IEEE Transactions on Antennas and Propagation, vol. 56, no. 4, pp. 999-1007, 2008.

[6] W. Y. Nie and Z. G. Wang, "Efficient computation of wideband RCS using singular value decomposition enhanced improved ultrawideband characteristic basis function method," International Journal of Antennas and Propagation, vol. 2016, Article ID 6367205, 6 pages, 2016.

[7] C. S. Park, Y. R. Jeong, I. P. Hong et al., "Analysis of curved frequency selective surface for radome using characteristic basis function method," in 2016 10th European Conference on Antennas and Propagation (EuCAP), pp. 1-4, Davos, Switzerland, April 2016.

[8] W. C. Chew, E. Michielssen, J. M. Song, and J. M. Jin, Fast and Efficient Algorithms in Computational Electromagnetics, Artech House, Norwood, MA, USA, 2001.

[9] FEKO, “Overview of FEKO," http://www.feko.info. 


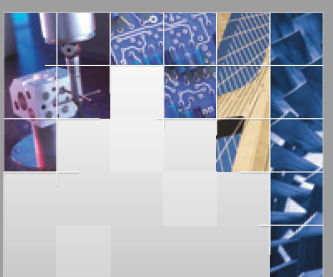

\section{Enfincering}
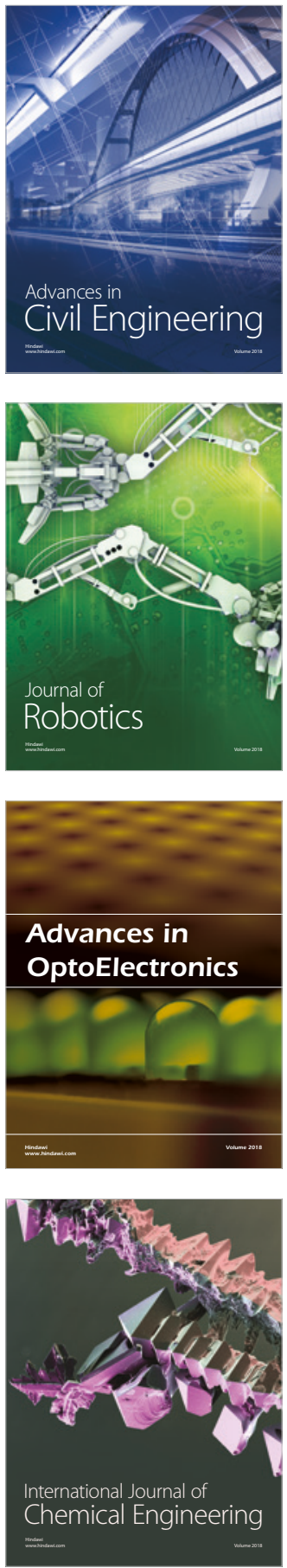

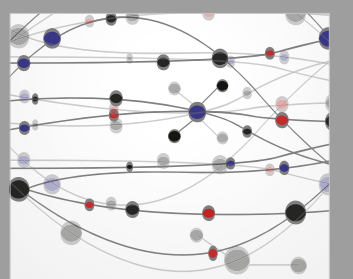

\section{Rotating \\ Machinery}

The Scientific World Journal

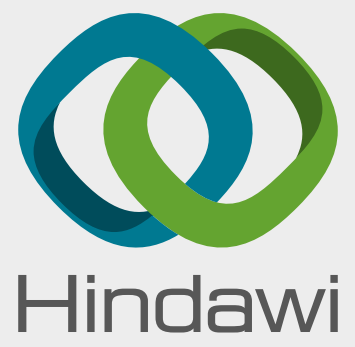

Submit your manuscripts at

www.hindawi.com
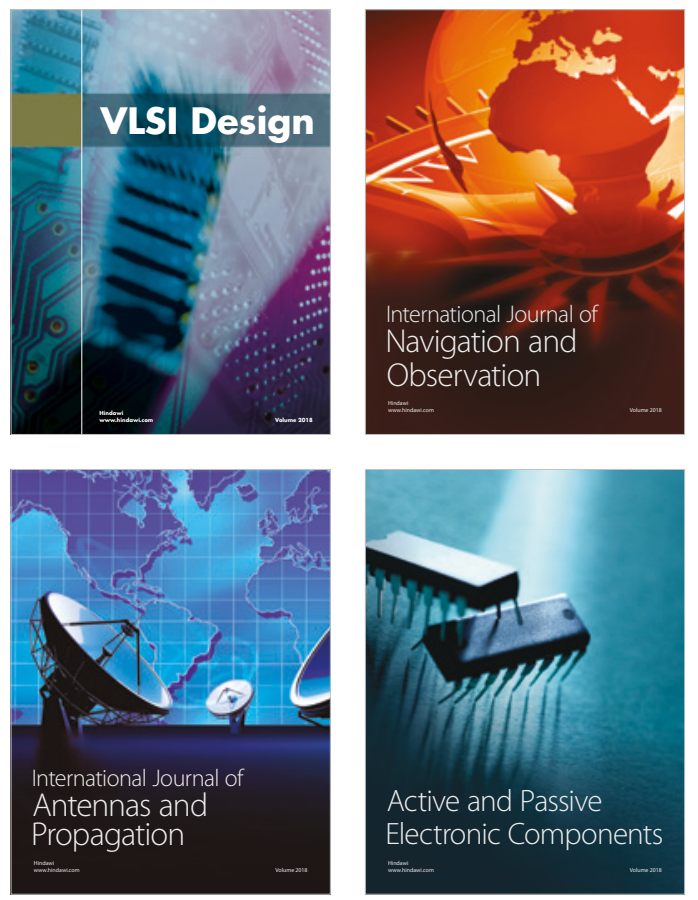
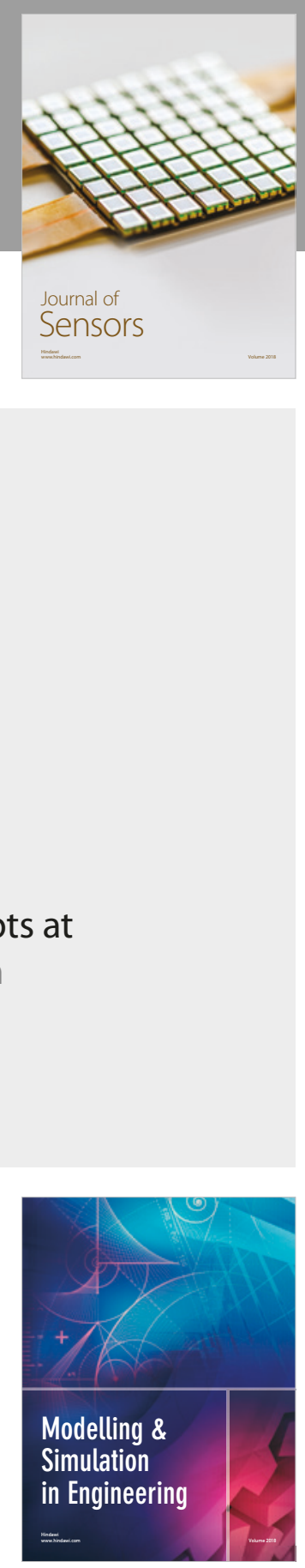

\section{Advances \\ Multimedia}
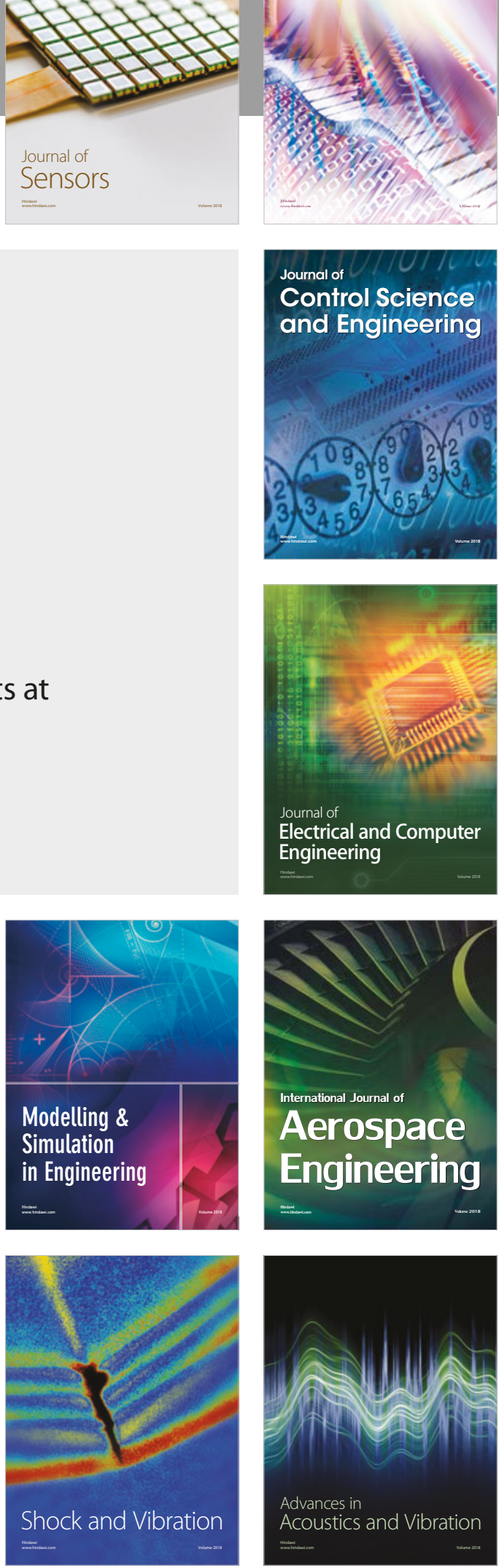